ARTICLE

\title{
Ultrafast femtosecond pressure modulation of structure and exciton kinetics in 2D halide perovskites for enhanced light response and stability
}

Chunpeng Song ${ }^{1}$, Huanrui Yang ${ }^{1}$, Feng Liu $^{1} \&$ Gary J. Cheng $\mathbb{B}^{1,2,3 凶}$

The carriers' transportation between layers of two-dimensional (2D) perovskites is inhibited by dielectric confinement. Here, for the first time, we employ a femtosecond laser to introduce ultrafast shock pressure in the range of $0 \sim 15.45 \mathrm{GPa}$ to reduce dielectric confinement by modulating the structure and exciton dynamics in a perovskite single crystal (PSCs), e.g. (F$\mathrm{PEA})_{2} \mathrm{Pbl}_{4}$ (4-fluorophenethylammonium, F-PEA). The density functional theory (DFT) simulation and experimental results show that the inorganic framework distortion results in a bandgap reduction. It was found that the exciton-optical phonon coupling and free excitons (FEs) binding energy are minimized at $2.75 \mathrm{GPa}$ shock pressure due to a reduction in dielectric confinement. The stability testing under various harsh light and humid thermal conditions shows that femtosecond laser shocking improves the stability of (F-PEA)2Pbl4 PSCs. Femtosecond laser shock processing provides a new approach for regulating the structure and enhancing halide perovskite properties.

\footnotetext{
${ }^{1}$ The Institute of Technological Sciences, Wuhan University, Wuhan, China. ${ }^{2}$ Brick Nanotechnology Center, Purdue University, West Lafayette, IN, USA

${ }^{3}$ School of Industrial Engineering, Purdue University, West Lafayette, IN, USA. ${ }^{\star}$ email: gjcheng@purdue.edu
} 
T he 3D organic-inorganic halide perovskite as lightabsorbing materials for photovoltaic devices has attracted extensive attention with certified power conversion efficiencies (PCE) exceeding $23 \%$ in just over a decade ${ }^{1-3}$. However, perovskite's long-term stability remains a pressing challenge. $2 \mathrm{D}$ perovskite is one type of quantum-well (QW)-like materials representing $R_{2} A_{n-1} B_{n} X_{3 n+1}$, where $R$ and $A$ are organic cations, $\mathrm{B}$ is a metal cation, $\mathrm{X}$ is a halide, and $\mathrm{n}$ value is metal cation layers thickness. The $\mathrm{n}$ value determines the quantum and the dielectric confinement degree ${ }^{4}$, and the $R$ influences the stability ${ }^{5}$ as well as flexibility ${ }^{6}$, which enables the modulation of the performance of $2 \mathrm{D}$ perovskite photoelectric to the greatest extent. Therefore, 2D perovskites have many promising applications in light-emitting diodes $(\mathrm{LED})^{7}$, detectors ${ }^{8}$, and lasers ${ }^{9}$ owing to excellent light, thermal, and humid thermal stability compared to their $3 \mathrm{D}$ counterparts ${ }^{10-12}$.

The large size of the organic spacer cations (R) for 2D perovskite permits absorption in the visible range significantly lower than that of its $3 \mathrm{D}$ counterpart ${ }^{13,14}$. In other words, a large bandgap in 2D perovskites inhibited light utilization at 500-780 $\mathrm{nm}$. Besides, the tremendous exciton binding energy in $2 \mathrm{D}$ perovskites is mainly attributed to dielectric confinement ${ }^{15}$. Several hundred meV binding energy is difficult to separate for the free carriers collected by the electrode without an external drive ${ }^{16}$. Regulating the structure of 2D perovskite and exciton transport dynamics is a key to improve its efficiency. Because of the soft lattice nature and the flexibility of sizeable organic spacer cations, the pressure control in the $2 \mathrm{D}$ perovskite structure becomes a vital means ${ }^{17,18}$. Currently, most of the research focuses on structure modulation via diamond anvil cell (DAC), while the kinetics of exciton transport after pressure is of little concern ${ }^{19,20}$. Ultrafast laser shocking has a much shorter mechanical interaction time that differs from DAC in maintaining the metastable state of the structure, while the structure after DAC is a reversible process and returns to its original condition after processing, which makes it difficult to control the structure and properties. In addition, the ultrashort energy deposition time limits the thermal energy diffusion away from the laser-shock zone, which is beneficial to reduce the irreversible damage to the material caused by heat. Controlling the plastic deformation generated by local residual compressive stress caused by the pulse laser-shock wave propagating in the material is beneficial to realize the control of local defects ${ }^{21}$. Simultaneously, the laser shocking pressure can be tuned from one to hundreds of gigapascals with selected pulse duration, energy density, and ablator ${ }^{22,23}$. Besides, ultrashort energy deposition time and the adjustability of pulse laser beam size promise to accomplish laser-shock processing in a short time. Thus, femtosecond laser shocking has unparalleled advantages over DAC in large-area controllable processing. The application of the shock pressing with a nanosecond pulse laser has been applied in residual stress regulation and grain size optimization of perovskite thin films ${ }^{24}$, nanolithography of metallic thin films ${ }^{25}$, strain engineering ${ }^{26}$, and patterned transformation ${ }^{27}$. However, the ultrashort energy deposition time and wide ranges of pressure control in femtosecond laser-shock processing will bring many different conditions for structure changes and exciton kinetics in semiconductors and fully utilize the potential advantages of ultrafast shock processing.

In this work, we investigate the structure and exciton kinetics of (F-PEA $)_{2} \mathrm{PbI}_{4}$ PSCs under femtosecond laser shock in the range of $0-15.45 \mathrm{GPa}$. To our knowledge, it is the first application of pulsed femtosecond laser shocking in halide perovskites as well as the relationship between crystal structure and exciton kinetics of ultrafast pressure variation in perovskite was studied systematically, which photodetector device performance have been clear as well. First, the $150 \mathrm{meV}$ reduction of the bandgap of
(F-PEA) ${ }_{2} \mathrm{PbI}_{4}$ PSCs after ultrafast pressure rise to $15.45 \mathrm{GPa}$, appearing a critical pressure at $2.75 \mathrm{GPa}$. The crystal structure and DFT simulation analysis shows that the change of $\mathrm{Pb} 6 p$ and I $5 p$ orbitals overlap caused by the distortion of the inorganic frameworks is the main reason for the band structure change. Besides, the binding energy and the exciton-optical phonon coupling of FEs are weakened to promote exciton separation. The balanced electron/hole effective mass and femtosecond timescale shock wave restrict defects, result in carrier transport and collection enhancement, which boosts photocurrent from 75 to 403 $\mathrm{nA}$ in an (F-PEA) $)_{2} \mathrm{PbI}_{4}$ PSCs photodetector. Finally, the stability testing under various harsh light and humid thermal conditions shows that ultrafast pressure improves the stability of (F$\mathrm{PEA})_{2} \mathrm{PbI}_{4}$ PSCs by fine-tuning the inorganic frameworks. Therefore, ultrafast laser shocking provides a facile and robust way to modulate the structure and exciton dynamics of perovskites and enhance their properties.

\section{Results}

Femtosecond laser shocking ultrafast pressure process. Most 2D Perovskites are malleable because of their soft nature lattice and flexible organic cations. The relationship between crystal structure and photoluminescence (PL) emission of 2D perovskite compressive by DAC pressure has received significant attention 17,28 . However, the local plastic deformation resulting from femtosecond laser shocking has more advantages in strain control than elastic deformation induced by DAC pressure processing. The schematic of the femtosecond laser-shock deformation process of 2D perovskite is shown in Fig. 1a. A high energy density femtosecond laser beam was focused onto the graphite surface, generating plasma, inducing a pulse pressure as a result of the plasma recoil momentum. Transparent dielectric glass restricted the hot plasma adiabatic expansion, resulting in an ultrafast shock pressure toward perovskites ${ }^{29}$. Besides, aluminum foil with high thermal conductivity acted as the thermal conduction and isolation layers, avoiding tension stress and pollution of perovskite surfaces caused by direct plasma heating. The $2 \mathrm{D}$ perovskites were subjected to femtosecond laser-induced ultrafast pressure perpendicular to the inorganic frameworks, which induces two deformation modes to take place, i.e., the reduction of interlayer distance and distortion of the inorganic frameworks. Ultrafast pressure enhances benzene dipole interaction and $\pi-\pi$ conjugate, and promotes carriers' transport, while the distortion of the inorganic frameworks reduced the long-distance order of 2D perovskite. The regulation of these two competitive processes has been a primary method to modulate the optoelectronic properties and structure of $2 \mathrm{D}$ perovskite.

The ultrafast pressure is controlled by laser power density, ablator, and pulse duration ${ }^{30}$. The maximum ultrafast pressure and shock wave velocity were calculated, which increased with the laser energy increasing and decreasing pulse duration (Fig. $1 \mathrm{~b}$ and calculation). Simultaneously, the shock wave is spherical expansion and penetration depth up to dozens of millimeters ${ }^{31}$. The thickness of (F-PEA) ${ }_{2} \mathrm{PbI}_{4}$ PSCs has only $15 \mu \mathrm{m}$ (Supplementary Table 1), which far less than the penetration depth of the shock wave. As a consequence, we ignore the shock wave decay in perovskite. Shock wave induced by femtosecond travels at a speed of thousands of meters per second through the flyer (aluminum foil) to reach the perovskite surface. The femtosecond energy deposition time limits the diffusion of thermal energy away from the laser-shock zone. Meanwhile, due to the rapid cooling of plasma, the duration of the shock wave is approximately equal to the pulsed laser duration. According to Ballard model ${ }^{32}$, the residual compressive stress is positively correlated with the pulse duration, which is the highest at (F-PEA) ${ }_{2} \mathrm{PbI}_{4}$ PSCs surface. 

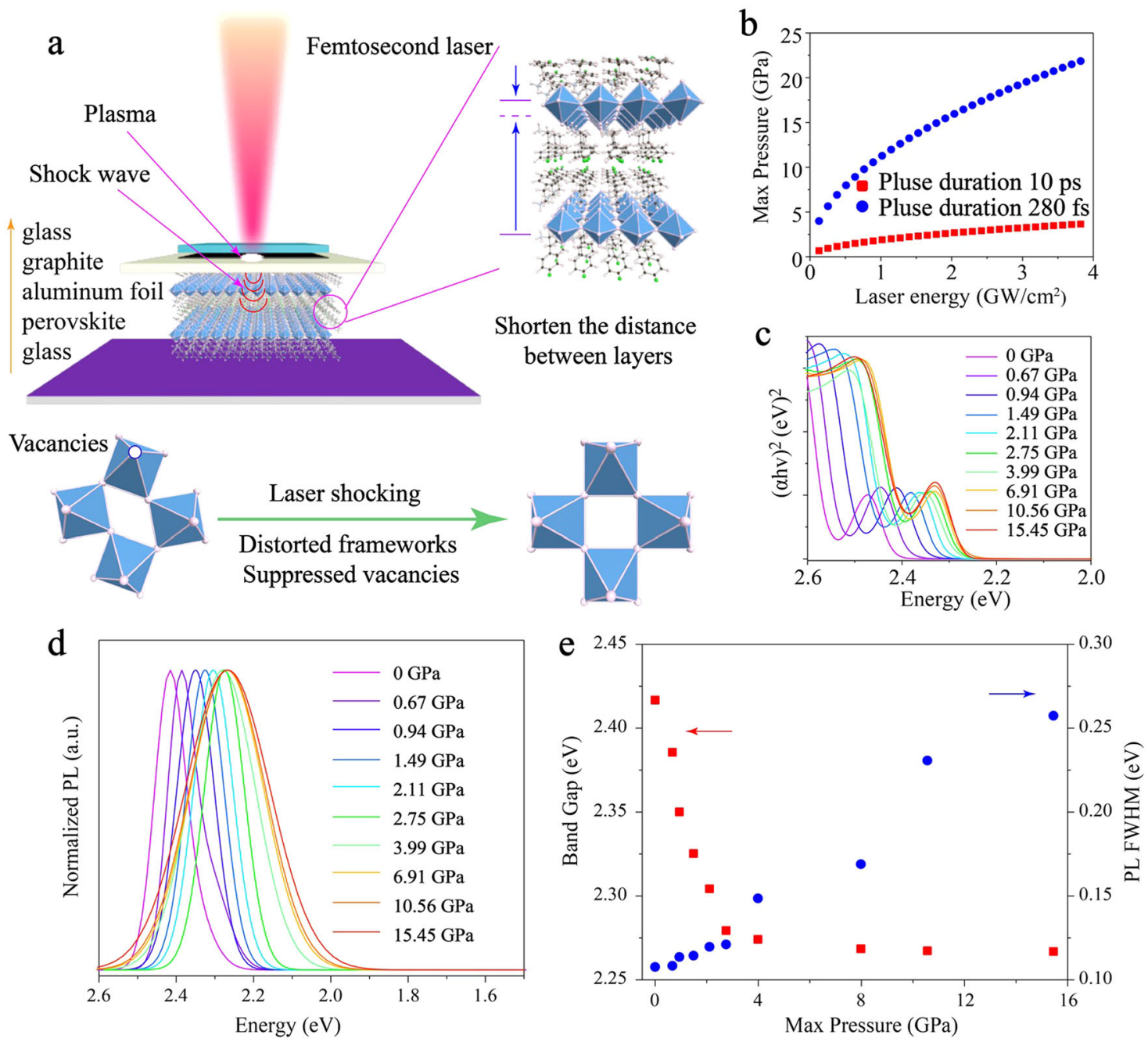

Fig. 1 The bandgap modulation of (F-PEA) ${ }_{2} \mathbf{P b l}_{4}$ PSCs via femtosecond laser shocking. a Schematic of the laser-shock deformation process of (F$\mathrm{PEA})_{2} \mathrm{Pbl}_{4} \mathrm{PSC}$. $\mathbf{b}$ The relationship between laser energy density and maximum laser shocking pressure. c Tauc curves obtained from ultraviolet-visible absorption spectra reveal bandgap values variation with ultrafast pressure. $\mathbf{d}$ photoluminescence spectra under ultrafast pressure. e According to the Tauc curve, the bandgap decreases with ultrafast pressure, while the full-width at half-maximum (FWHM) of photoluminescence (PL) spectra is the opposite.

Besides, the vacancy defects on the halide perovskite surface are mainly caused by ion migration, which is suppressed by residual compressive stress ${ }^{33}$. Ion migration occurs on a timescale from seconds to minutes ${ }^{34}$, whose timescale is orders of magnitude longer than the duration of the shock wave. As a result, the compression and decompression processes were completed before the defects were diffused. Femtosecond laser ultrafast shock wave compression and decompression manage the local compressive strain of $2 \mathrm{D}$ perovskite 21,35 . Accordingly, ultrashort mechanical interaction time of pulsed laser shocking has incomparable advantages in material structure and performance regulation.

Ultrafast pressure balance band structure. To investigate the structure and performance of $2 \mathrm{D}$ perovskite after femtosecond laser shocking,(F-PEA) ${ }_{2} \mathrm{PbI}_{4}$ PSCs were fabricated by a cooling solution method ${ }^{36}$. The crystal structure and morphology were characterized by powder X-ray diffraction (PXRD) and field emission scanning electron microscopy (FE-SEM) mapping as well as energy-dispersive X-ray spectroscopy (EDS), as shown in Supplementary Figs. 2-5. The crystal orientation, distribution, and proportion of elements demonstrate that high-quality (F$\mathrm{PEA})_{2} \mathrm{PbI}_{4}$ PSCs had been synthesized. Figure 1c illustrated the bandgap variation of (F-PEA $)_{2} \mathrm{PbI}_{4}$ PSCs under ultrafast shock wave pressure. With the gradual increase of pressure, the perovskite absorption spectrum and photoluminescence (PL) spectrum are redshift. The asymmetric emission spectrum, which was delivered from trapped states at atmospheric pressure, subsequently disappeared with the increased ultrafast pressure (Fig. 1d). Significant Stokes shift cannot be observed, indicating that nonradiative recombination energy loss is tiny with ultrafast pressure. By comparing the bandgap and PL full-width at halfmaximum (FWHM), we found that the bandgap reduced by 150 $\mathrm{meV}$ as pressure increase from 0 to $2.75 \mathrm{GPa}$, while on the contrary, the PL FWHM increased from 0.10 to $0.12 \mathrm{eV}$ (Fig. 1e). Further increase of pressure from $2.75 \mathrm{GPa}$ on (F-PEA) ${ }_{2} \mathrm{PbI}_{4}$ PSCs would cause irreversible plastic deformation, similar to the amorphous of other perovskites under high pressure ${ }^{37,38}$. We examined the bandgap change over time to determine the (F$\mathrm{PEA})_{2} \mathrm{PbI}_{4}$ PSCs structure's stability (Supplementary Fig. 7). We found that the bandgap remained stable for $144 \mathrm{~h}$ after applying the femtosecond shock, and the absorption bandgap could not recover. Therefore, we speculate that the structural distortion of perovskite after femtosecond laser shock can remain stable for at least $144 \mathrm{~h}$. The ultrafast femtosecond pressure modulation was also applied on $(\mathrm{PEA})_{2} \mathrm{PbI}_{4}$ PSCs. As shown in an ultraviolet- 

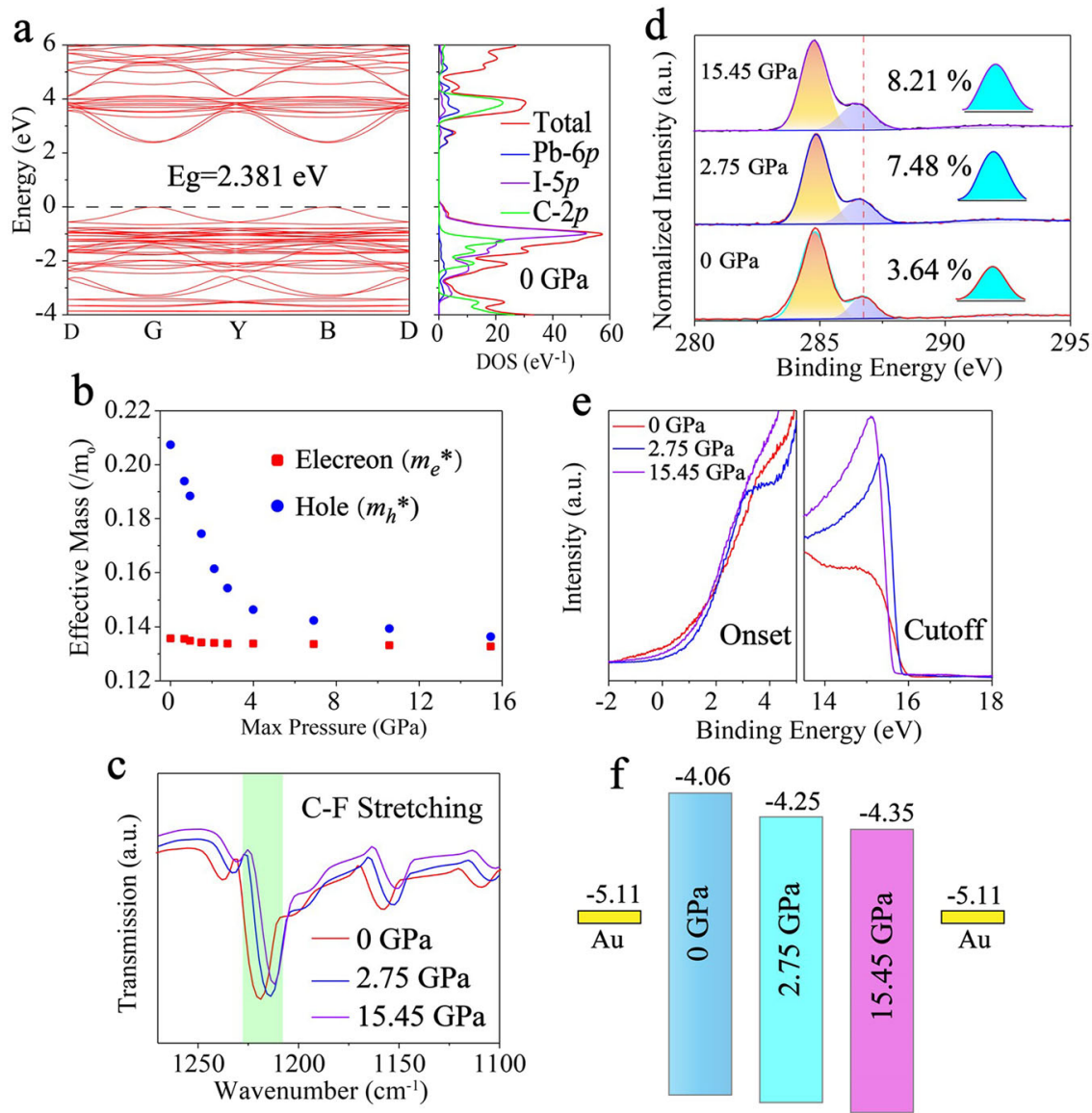

Fig. 2 Ultrafast pressure balance band structure of (F-PEA) ${ }_{2} \mathbf{P b I}_{\mathbf{4}} \mathbf{P S C}$. a Density functional theory simulated the band structure and density of states (DOS) without ultrafast pressure, b Simulated perovskite effective mass as a function of ultrafast pressure. c Fourier transform infrared spectra of (F$\mathrm{PEA})_{2} \mathrm{Pbl}_{4} \mathrm{PSC}$. d X-ray photoelectron spectroscopy spectra at three representative ultrafast pressures of (F-PEA) ${ }_{2} \mathrm{Pbl}_{4} \mathrm{PSC}$. e Ultraviolet photoelectron spectroscopy spectra of onset and cutoff for (F-PEA) ${ }_{2} \mathrm{Pbl}_{4} \mathrm{PSCs}$. $\mathbf{f}$ Schematic diagram of band alignment of (F-PEA) ${ }_{2} \mathrm{Pbl}_{4} \mathrm{PSCs}$ relative to Au under ultrafast pressure.

visible (UV-Vis) absorption spectrum (Supplementary Fig. 8), a similar behavior with (F-PEA) ${ }_{2} \mathrm{PbI}_{4}$ PSCs was discovered, indicating femtosecond laser-shock-induced structural modulation can be extended to other similar materials.

The band structure balance between the layers reduces the carrier transmission barrier and the carrier accumulation at the interface, which is beneficial to both carrier transmission and collection. The band structure and density of states (DOS) for the DFT simulated (F-PEA) ${ }_{2} \mathrm{PbI}_{4}$ PSCs after ultrafast pressure were summarized in Fig. 2a and Supplementary Fig. 18. Partial density of states (PDOS) revealed that the conduction band minima $(\mathrm{CBM})$ and valence band maxima $(\mathrm{VBM})$ of $(\mathrm{F}-\mathrm{PEA})_{2} \mathrm{PbI}_{4} \mathrm{PSCs}$ came from $\mathrm{Pb} 6 p$ and I $5 p$ orbitals, respectively. Nevertheless, the organic cation was not able to make a direct contribution. The (F$\mathrm{PEA})_{2} \mathrm{PbI}_{4}$ PSCs is a direct bandgap semiconductor with a calculated bandgap of $2.381 \mathrm{eV}$. The bandgap decreases rapidly to $2.215 \mathrm{eV}$ at $2.75 \mathrm{GPa}$ and continues decreasing at a slower rate under shock pressure from 2.75 to $15.45 \mathrm{GPa}$ (Supplementary Fig. 19), which matched the experimental results very well. The effective mass could be evaluated by $m_{e, h}{ }^{*}=\hbar /\left[\partial^{2} \varepsilon(\mathbf{k}) / \partial \mathbf{k}^{2}\right]$ where $\varepsilon(\mathrm{k})$ is the eigenvalue of the wave vector $\mathrm{k}$ along the transport direction, and $\hbar$ is Planck's constant. The effective mass of electrons $\left(m_{e}{ }^{*}\right)$ and holes $\left(m_{h}{ }^{*}\right)$ with pressure were summarized in Fig. 2 b. The $m_{h}{ }^{*}$ decreased with the pressure and finally approached $m_{e}^{*}$ at $15.45 \mathrm{GPa}^{34}$. The balanced electron/hole effective mass promotes carriers' transport and collection, owing to local residual compressive stress regulation as a result of ultrafast compress and decompress via femtosecond laser shocking. Moreover, we have studied the molecular interaction of perovskite in the Fourier transform infrared (FTIR) spectrum. With femtosecond laser shock, C-F stretching and benzene vibration frequencies are enhanced, as shown in Fig. 2c and Supplementary Fig. 20. X-ray photoelectron spectroscopy (XPS) of (F-PEA) $)_{2} \mathrm{PbI}_{4}$ PSCs at atmospheric pressure shown that three C $1 s$ peaks: C-C $(284.79 \mathrm{eV}), \mathrm{C}-\mathrm{N}(286.72 \mathrm{eV})$, and $\pi-\pi$ $(292.24 \mathrm{eV})^{3}$ (Fig. 2d). The increased pressure weakens the $\mathrm{C}-\mathrm{N}$ binding energy, while the $\mathrm{C}-\mathrm{C}$ remains unchanged. The $\mathrm{N} 1 \mathrm{~s}$ peak was blueshift and the $\mathrm{Pb} 4 f$ peak was redshift, as shown in Supplementary Fig. 21. This contrary change confirmed that the organic cation indirectly affecting the band structure through the $\mathrm{N}-\mathrm{H}$...I hydrogen bonding ${ }^{39}$. The relative peak area of $\pi-\pi$ interaction as a function of pressure announced that dipole interaction between layers raised, promoting carriers to pass through organic layers ${ }^{40}$. The work function and VBM were obtained by ultraviolet photoelectron spectroscopy (UPS) ${ }^{41}$, as delineated in Fig. 2e. The energy between the VBM and the Fermi level as well as the work function enlarged with ultrafast shock pressure. The CBM and VBM of (F-PEA) ${ }_{2} \mathrm{PbI}_{4}$ PSCs shifted down to the deep energy level (Fig. 2f). Also, the CBM was gradually aligned with the $\mathrm{Au}$, reducing electronic potential barriers between (F-PEA) ${ }_{2} \mathrm{PbI}_{4}$ PSCs.

The source of band structure regulation. The crystal structure of the perovskite directly affects its properties and the management 

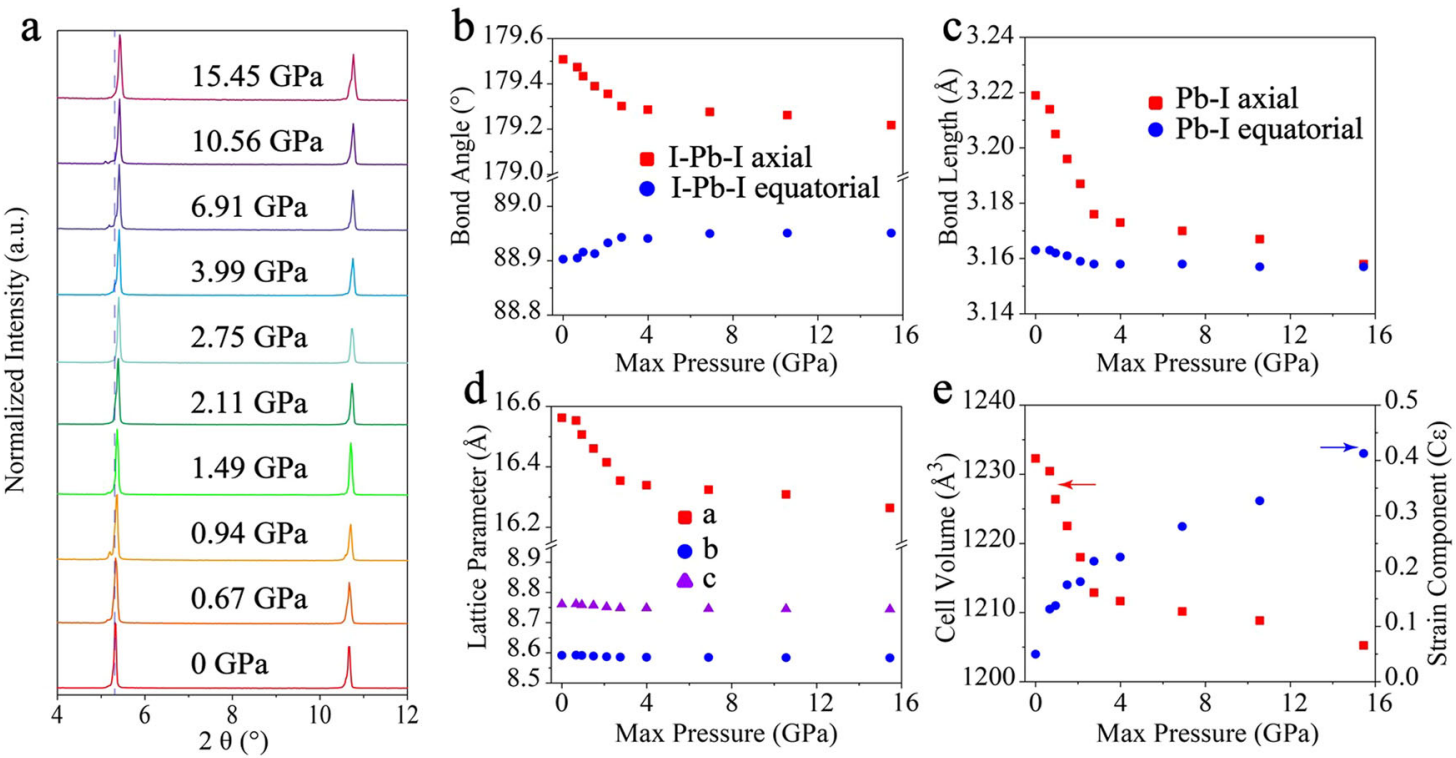

Fig. 3 Ultrafast pressure regulation lattice of (F-PEA) $\mathbf{P b l}_{\mathbf{4}} \mathbf{P S C}$. a With the increase of ultrafast pressure, the powder X-ray diffraction spectrum gradually redshifts for (F-PEA) ${ }_{2} \mathrm{Pbl}_{4}$ PSCs. $\mathbf{b}$ Ultrafast pressure-dependent I-Pb-I bond angles and $\mathbf{c}$ Pb-I bond lengths. Obviously, the inorganic frameworks distorted with pressure can be observed. d Unit cell parameters of (F-PEA) ${ }_{2} \mathrm{Pbl}_{4}$ PSCs as a function of pressure. e Unit cell volumes evolution and Williamson-Hall strain component of (F-PEA) ${ }_{2} \mathrm{Pbl}_{4} \mathrm{PSCs}$ with increasing ultrafast pressure.

of the (F-PEA) ${ }_{2} \mathrm{PbI}_{4}$ PSCs lattice has become an important method to regulate its photoelectric performance. Crystallographic data for (F-PEA) $)_{2} \mathrm{PbI}_{4}$ PSCs were summarized in Supplementary Table 1, in which (F-PEA) ${ }_{2} \mathrm{PbI}_{4}$ PSCs are monoclinic systems with $\mathrm{P} 2{ }_{1} / \mathrm{c}(\# 14)$ group ${ }^{42}$. Figure 3 a shows the PXRD pattern with pressure, the (001) peak of (F-PEA) ${ }_{2} \mathrm{PbI}_{4}$ PSCs redshifts as the pressure. There is no new crystal peak within the ultrafast pressure range of our experiment, indicating that the irreversible phase transition does not occur after ultrafast compression and decompression. Figure $3 b$, c summarizes the relationship between bond Angle and bond length with pressure. The bond angle and bond length change from 0 to $2.75 \mathrm{GPa}$ significantly exceeded that from 2.75 to $15.45 \mathrm{GPa}$. Moreover, the axial shortening of the $\mathrm{Pb}-\mathrm{I}$ bond length beyond that of the equatorial, consistent with the $\mathrm{I}-\mathrm{Pb}-\mathrm{I}$ angle. The a axis of (FPEA) ${ }_{2} \mathrm{PbI}_{4}$ PSCs unit cell decreased by about $0.3 \AA$, while the $b$ and $c$ axes remain unchanged, indicating that the pressure is mainly in the axial compression (Fig. 2d). The reduction of unit cell lattice constants was more significant than the bond length, indicating the organic cation is more compressible than the inorganic frameworks. The unit cell volume also downturn appropriately, and the inflection at $2.75 \mathrm{GPa}$ marked the compression limit of (F-PEA) ${ }_{2} \mathrm{PbI}_{4}$ PSCs. Williamson-Hall plot was used to estimate the stress in the lattice ${ }^{43,44}$. The slope of the plot represented the strain constant $(\mathrm{C} \varepsilon)$, but it was not to be taken strictly, which performed the diversity of strain to some extent (Supplementary Fig. 22). $C \varepsilon$ as a function of max pressure was demonstrated in Fig. 2e. The stress accumulates continuously in the lattices and is altered at $2.75 \mathrm{GPa}$ as well. High lattice strain under $15.45 \mathrm{GPa}$ weakens the long-range order of (F-PEA) ${ }_{2} \mathrm{PbI}_{4}$ PSCs, conformed with PL peak broadening.

Modulation of exciton kinetics and defects. The separation of photogeneration excitons is the key to improve the performance of 2D perovskite optoelectronic devices. The exciton binding energy of $2 \mathrm{D}$ perovskites is in the range of $100-500 \mathrm{meV}$, far beyond their $3 \mathrm{D}$ counterparts ${ }^{45,46}$. The exciton properties of (F-PEA) ${ }_{2} \mathrm{PbI}_{4} \mathrm{PSCs}$ with ultrafast pressure were investigated in detail by temperaturedependent PL spectra, as depicted in Fig. 4 and Supplementary
Fig. 23. The peaks at $1.94 \mathrm{eV}$ and $2.37 \mathrm{eV}$ attribute to shallow trap excitons and FEs, respectively ${ }^{16,17,47}$. Temperature-dependent PL peak width broadening of FEs was fitted with the Boson model: ${ }^{48}$

$$
\Gamma(T)=\Gamma_{0}+\frac{\Gamma_{o p}}{\exp \left(\hbar w_{o p} / k_{\mathrm{B}}\right)-1}
$$

Where $\Gamma_{0}$ represents the inhomogeneous broadening, $\Gamma_{o p}$ is the exciton-optical phonon contribution and $\hbar w_{o p}$ the optical phonon energy. Compared with (F-PEA) ${ }_{2} \mathrm{PbI}_{4}$ PSCs at atmospheric pressure, the $2.75 \mathrm{GPa}$ femtosecond laser shocking resulted in inhomogeneous broadening and exciton-optical phonon as well as optical phonon energy, decreased to $81.1 \pm 1.3,15.5 \pm 1.1$, and 32.6 $\pm 2.9 \mathrm{meV}$, respectively (Fig. $4 \mathrm{a}$ ). The fundamental reason is that the enhanced dipole interaction and the reduced dielectric confinement ${ }^{49}$. With the highest ultrafast pressure of $15.45 \mathrm{GPa}$, the strain accumulated with the disordered electronic structure in (F$\mathrm{PEA})_{2} \mathrm{PbI}_{4}$ PSCs, leading to optical phonon scattering enhancement. Inhomogeneous broadening, exciton-optical phonon, and optical phonon energy had been up to $96.2 \pm 4.6,21.7 \pm 1.8$, and $39.8 \pm 3.7 \mathrm{meV}$, respectively. Their value is still below that at $0 \mathrm{GPa}$, owing to a reduction in dielectric confinement caused by the $\pi-\pi$ enhancement. The temperature-dependent FEs binding energy $\left(E_{\mathrm{B}}\right)$ were appraised with the formula ${ }^{50}$ :

$$
I(T)=\frac{I_{0}}{1+A \exp \left(-E_{\mathrm{B}} / k_{\mathrm{B}} T\right)}
$$

in which $I_{0}$ and $k_{\mathrm{B}}$ are intensity at $0 \mathrm{~K}$ and Boltzmann constant. The $E_{\mathrm{B}}$ of (F-PEA) $)_{2} \mathrm{PbI}_{4}$ PSCs at $0 \mathrm{GPa}$ was $431 \mathrm{meV}$, which was down to $274 \mathrm{meV}$ at $2.75 \mathrm{GPa}$ and up to $342 \mathrm{meV}$ at $15.45 \mathrm{GPa}$ (Fig. $4 \mathrm{~b}$ ). Competition between the disorder of electronic structure and dipole interaction resulted in the minimum $E_{\mathrm{B}}$ at $2.75 \mathrm{GPa}$.

We extracted PL spectra at $80 \mathrm{~K}$ to investigate shallow trap excitons (at $1.94 \mathrm{eV}$ ), as depicted in Fig. 4c. Compared to the shallow trap excitons of (F-PEA) $)_{2} \mathrm{PbI}_{4}$ PSCs at $0 \mathrm{GPa}$, the value slightly decreased at $2.75 \mathrm{GPa}$, while it significantly increases at $15.45 \mathrm{GPa}$, indicating that the defects in perovskite reduce after proper pressure. Besides, pressure changed the energy transfer barrier of FEs and shallow trap excitons, also the inducer of shallow trap excitons diversity ${ }^{51}$. An apparent exciton emission 

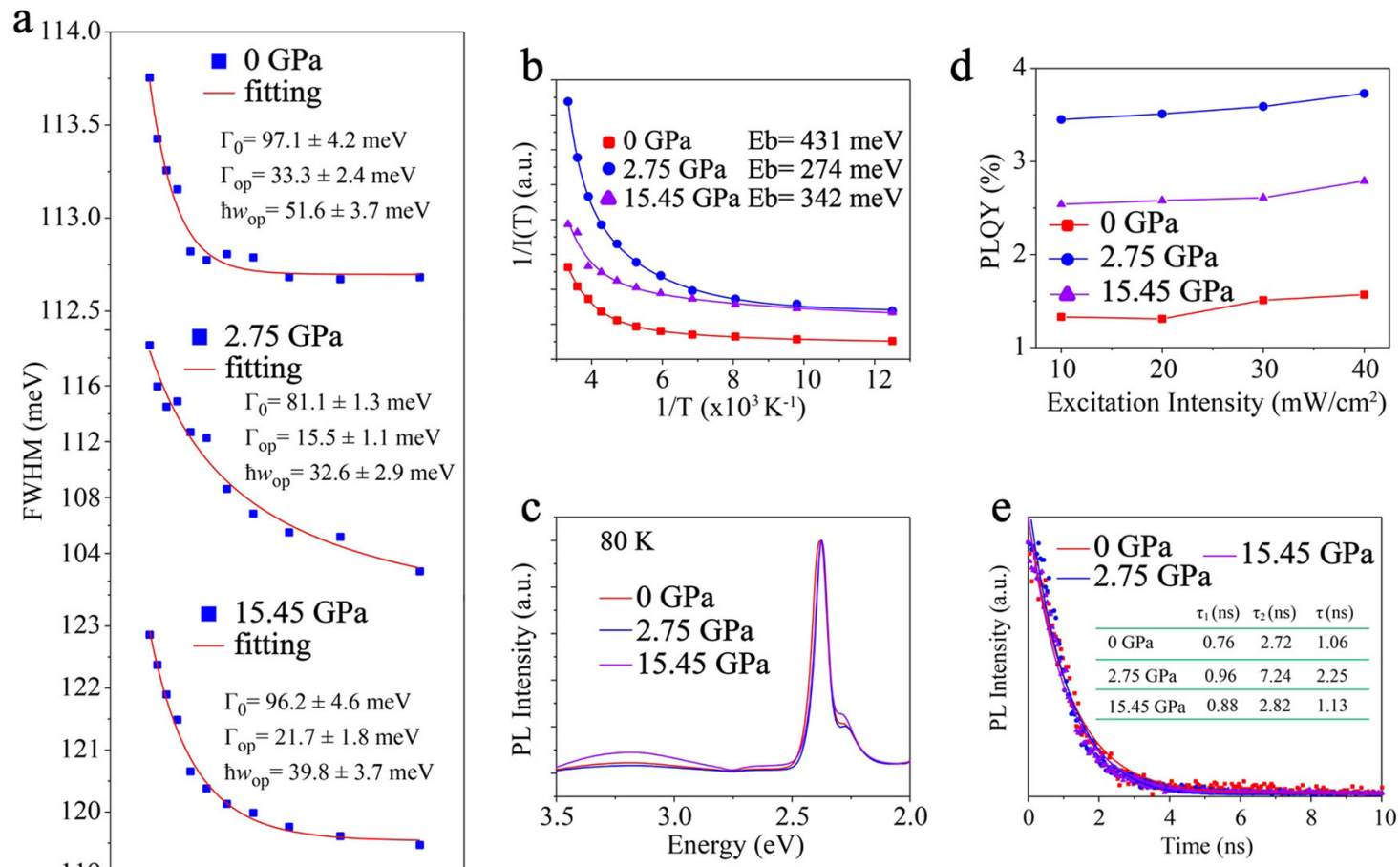

Fig. 4 Ultrafast pressure modulation exciton kinetics and defects of (F-PEA) $\mathbf{P b l}_{\mathbf{4}} \mathbf{P S C s}$. a Temperature-dependent photoluminescence (TRPL) fullwidth at half-maximum (FWHM) of (F-PEA) ${ }_{2} \mathrm{Pbl}_{4}$ PSCs. b Free excitons binding energy for (F-PEA)2Pbl4 PSCs by fitting temperature-dependent photoluminescence spectra. c Photoluminescence spectra of (F-PEA) ${ }_{2} \mathrm{Pbl}_{4} \mathrm{PSCs}$ with ultrafast pressure at $80 \mathrm{~K}$. d Photoluminescence quantum yields $(\mathrm{PLQY})$ in ( $\mathrm{F}-\mathrm{PEA})_{2} \mathrm{Pbl}_{4} \mathrm{PSCs}$ as a function of femtosecond laser shock for several light-excitation intensities. e Carriers lifetime of (F-PEA) ${ }_{2} \mathrm{Pbl}{ }_{4} \mathrm{PSCs}$ obtained from TRPL.

$(3.21 \mathrm{eV})$ peak appeared at $80 \mathrm{~K}$ and disappeared at $168 \mathrm{~K}$ only after the ultrafast pressure of $15.45 \mathrm{GPa}$, since exciton-phonon scattering enhances exciton restriction and benzene dipole interaction. Photoluminescence quantum yields (PLQY) for several excitation intensities were used to determine the regulation of (F-PEA) $)_{2} \mathrm{PbI}_{4}$ PSCs by femtosecond laser shock (Fig. 4d). Under air pressure, 1.3\% PLQY was obtained, which was much the same as that in previous reports of $2 \mathrm{D}$ perovskite ${ }^{12}$. With increasing pressure, PLQY was risen to $3.5 \%$ at $2.75 \mathrm{GPa}$, indicating reduced nonradiative losses. PLQY decreased to $2.6 \%$ at $15.45 \mathrm{GPa}$, resulting from excessive pressure destroying the long-range order of perovskite, which is consistent with PL broadening. The carrier's lifetime was obtained by fitting the time-resolved photoluminescence (TRPL) spectrum with the 2constant exponential decay function ${ }^{24}$. We characterized the intensity dependence TRPL and found that the lifetime reached the minimum at the excitation intensity of $300 \mathrm{~mW} / \mathrm{cm}^{2}$, as shown in Supplementary Fig. 24 and Supplementary Table 6-8. Therefore, TRPL with an excitation intensity of $300 \mathrm{~mW} / \mathrm{cm}^{2}$ was used to demonstrate exciton quenching lifetime $\left(\tau_{1}\right)$ and radiation recombination lifetime $\left(\tau_{2}\right)$. The exciton-exciton annihilation lifetime of (F-PEA) ${ }_{2} \mathrm{PbI}_{4}$ PSCs at $2.75 \mathrm{GPa}$ was just a little bit bigger than those at $0 \mathrm{GPa}$ and $15.45 \mathrm{GPa}$, respectively, while radiative recombination lifetime was near 2.5 times. The average lifetime $(\tau)$ was improved by $212 \%$, due to the reduced exciton binding energy and defect density. Also, band structure balance and $\pi-\pi$ enhancement of (F-PEA) $)_{2} \mathrm{PbI}_{4}$ PSCs were essential factors in carrier's lifetime.

Ultrafast pressure enhanced light response and robust structure. The structure and exciton dynamics of $2 \mathrm{D}$ perovskite directly affect its performance. Here, the photoelectric performance of (F-PEA) ${ }_{2} \mathrm{PbI}_{4}$ PSCs photodetector was characterized by $\mathrm{Au} /(\mathrm{F}-\mathrm{PEA})_{2} \mathrm{PbI}_{4}$ PSCs / Au structure. Photocurrent measurement was carried out on the (F-PEA) ${ }_{2} \mathrm{PbI}_{4}$ PSCs photodetector with an effective area of $5 \times 10^{-2} \mathrm{~mm}^{2}$ under $355 \mathrm{~nm}$ laser radiation (Intensity, $0.16 \mathrm{~mW} / \mathrm{cm}^{2}$ ). Figure $5 \mathrm{a}$ shows the current-voltage (I-V) curves of the devices made at various ultrafast pressure. Photocurrent made on $0 \mathrm{GPa}$ and $15.45 \mathrm{GPa}$ with $5 \mathrm{~V}$ bias at the same laser radiation was measured to be 75 $\mathrm{nA}$ and $257 \mathrm{nA}$, while its $403 \mathrm{nA}$ at $2.75 \mathrm{GPa}$, beyond $537 \%$ (Fig. 5b). The photocurrent enhancement is attributed to the much reduced FEs binding energy and suppressed excitonphonon coupling.

Stability is a prerequisite for the practical application of perovskite. we performed device stability measurements under operational conditions for (F-PEA $)_{2} \mathrm{PbI}_{4}$ PSCs photodetectors. As shown in Fig. $5 \mathrm{c}$, the photocurrent of the perovskite photodetector without pressure decreased rapidly to $67 \%$ of the initial photocurrent within $50 \mathrm{~h}$, and finally remained at $56 \%$ of the initial photocurrent after $500 \mathrm{~h}$. By comparison, perovskite was applied at ultrafast pressure of $2.75 \mathrm{GPa}$ and $15.45 \mathrm{GPa}$, maintaining $76 \%$ and $87 \%$ of the initial photocurrent after 500 h, respectively. XRD and XPS were characterized by the light and humid thermal stability of perovskite. The phase transition and decomposition of (F-PEA) ${ }_{2} \mathrm{PbI}_{4}$ PSCs were analyzed by differential scanning calorimetry (DSC) under the nitrogen atmosphere $^{52}$ (Supplementary Fig. 26). The fusion temperature of (F-PEA $)_{2} \mathrm{PbI}_{4}$ PSCs was found to be $518^{\circ} \mathrm{C}$ with an increase in enthalpy change under ultrafast pressure. The light stability of (FPEA) ${ }_{2} \mathrm{PbI}_{4}$ PSCs increases with the ultrafast pressure under a sunlight input for $10 \mathrm{~h}$, as explained by the $\mathrm{Pb}^{0} /\left(\mathrm{Pb}^{0}+\mathrm{Pb}^{2+}\right)$ increment (Fig. 5d) ${ }^{53}$. Figure 5e demonstrates the humid thermal stability of $(\mathrm{F}-\mathrm{PEA})_{2} \mathrm{PbI}_{4}$ PSCs over $10 \mathrm{~h}$ with relative humidity 


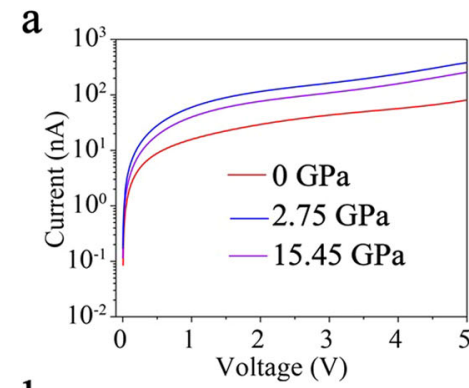

d

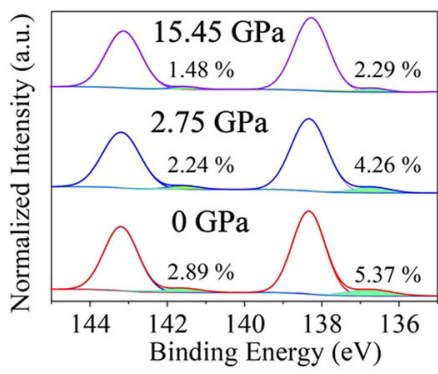

b

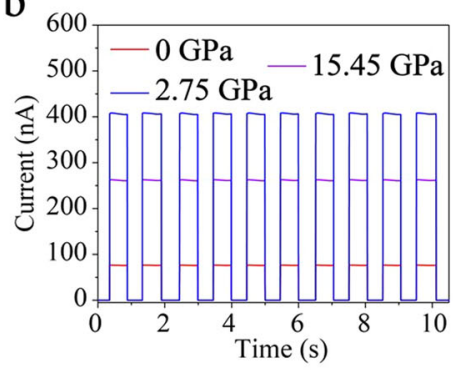

e

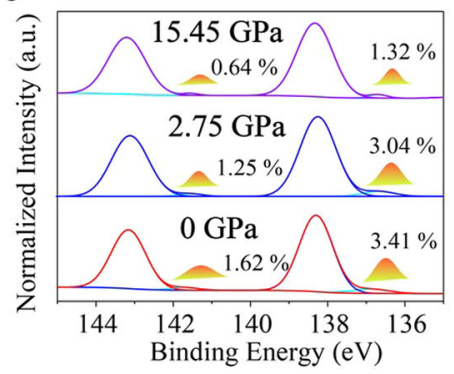

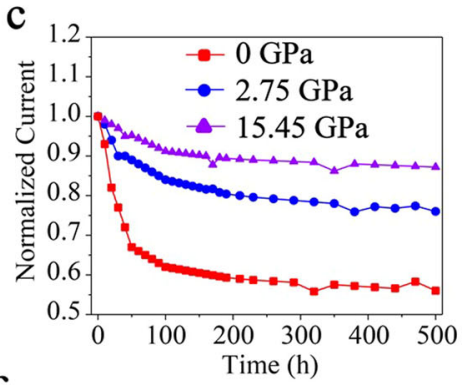

f

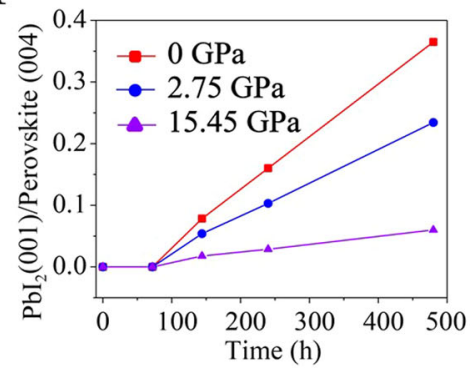

Fig. 5 Ultrafast pressure enhanced light response and robust structure for (F-PEA) $\mathbf{P}_{\mathbf{2}} \mathbf{P b}_{\mathbf{4}} \mathbf{P S C}_{\mathbf{2}}$ a Bias-dependent photocurrent of the (F-PEA) ${ }_{2} \mathrm{Pbl} \mathrm{P}_{4} \mathrm{PSCs}$

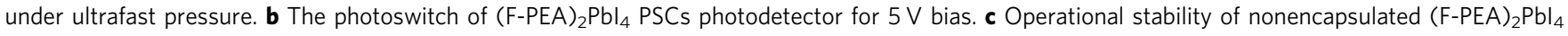
PSCs photodetectors, which were fabricated and tested in a room temperature and $85 \%( \pm 5 \%)$ relative humidity ambient atmosphere. Pb binding energy obtained from X-ray photoelectron spectroscopy to assessed $\mathbf{d}$ light stability (a sunlight input for $10 \mathrm{~h}$ ) and $\mathbf{e}$ humid thermal stability $\left(85^{\circ} \mathrm{C}, \mathrm{RH}=85 \%, 10\right.$ h) of $(\mathrm{F}-\mathrm{PEA})_{2} \mathrm{Pbl}_{4} \mathrm{PSCs}$. f Ratio of the $\mathrm{Pbl}_{2}\left(2 \theta=12.6^{\circ}\right.$ referred to $\left.<100>\right)$ and perovskite $\left(2 \theta=10.8^{\circ}\right.$ referred to $\left.<004>\right)$ main peaks over $500 \mathrm{~h}$.

(RH) $85 \%$ and temperature $85^{\circ} \mathrm{C}$. The $\mathrm{Pb}^{0} /\left(\mathrm{Pb}^{0}+\mathrm{Pb}^{2+}\right)$ was $5.03 \%$ without shock treatment, compared to $1.96 \%$ at $15.45 \mathrm{GPa}$ shock pressure. Figure $5 \mathrm{f}$ and Supplementary Fig. 27 show the air stability of (F-PEA) ${ }_{2} \mathrm{PbI}_{4}$ PSCs, which are measured at room temperature and $85 \%( \pm 5 \%)$ relative humidity. We found that the crystallization peak of lead iodide (001) appeared at $144 \mathrm{~h}$. The perovskite without femtosecond laser shock decomposed rapidly, while the perovskite decomposed significantly slower after applying ultrafast pressure. We attribute the stability improvement of (F-PEA) $)_{2} \mathrm{PbI}_{4}$ PSCs results from the optimization of lattice and band structure.

\section{Discussion}

In general, we introduce a pulsed laser to shock(F-PEA) $)_{2} \mathrm{PbI}_{4}$ PSCs for the first time and studied its structure and exciton dynamics in detail. The bandgap decreases by $150 \mathrm{meV}$ and remains stable with femtosecond laser shocking. DFT simulation and experimental results show that the reason for the decrease of the bandgap is the distortion of the inorganic frameworks. Through dipole interaction, the organic cation can distort the inorganic frameworks, and $\mathrm{N}-\mathrm{H} \cdots$ I hydrogen bonding indirectly affecting the band structure. By fitting the temperature-dependent PL spectrum, exciton-optical phonon coupling and optical phonon energy are $15.5 \pm 1.1 \mathrm{meV}$ and $32.6 \pm 2.9 \mathrm{meV}$ at $2.75 \mathrm{GPa}$. The FEs binding energy is $274 \mathrm{meV}$ at $2.75 \mathrm{GPa}$, much smaller than that at $0 \mathrm{GPa}$, owing to the $\pi-\pi$ enhancement and dielectric confinement reduction in (F-PEA) ${ }_{2} \mathrm{PbI}_{4}$ PSCs. The hole effective mass decreases while the electron effective mass is unchanged. The balanced electron/hole mass, as well as defect reduction, promote the carrier transport and collection, owing to local residual compressive stress regulation as a result of ultrafast compress and decompress via femtosecond laser shocking. The photocurrent increasing from 75 to $403 \mathrm{nA}$ of (F-PEA) ${ }_{2} \mathrm{PbI}_{4}$ PSCs photodetector, owing to carries lifetime increment and suppressed exciton-phonon coupling. More importantly, ultrafast laser shocking is shown to be a feasible method to modulate the structure and physical properties of perovskite, promising to improve electronic and optoelectronic performances of hybrid organic-inorganic halide perovskites and providing a critical solution to the stability as well.

\section{Methods}

Materials. 4-fluorophenethylammonium iodide (F-PEAI, purity > 99\%) purchased from Xi'an Polymer Light Technology Corp. Lead(II) oxide (PbO, purity > 99.999\%), Hydriodic acid (HI, 57 wt.\% in water), Hypophosphorous acid solution $\left(\mathrm{H}_{3} \mathrm{PO}_{2}, 50\right.$ wt.\% in water), and Hexane (purity $\left.>95 \%\right)$ purchased from Sigma-Aldrich. All materials are used as received.

(F-PEA) ${ }_{2} \mathrm{Pbl}_{4}$ and (PEA) ${ }_{2} \mathrm{Pbl}_{4}$ single-crystal growth. To fabricate (F-PEA) ${ }_{2} \mathrm{PbI}_{4}$ perovskite single crystal (PSCs), $44.6 \mathrm{mg} \mathrm{PbO}(0.2 \mathrm{mmol})$ and $106.8 \mathrm{mg}$ F-PEAI $(0.4 \mathrm{mmol})$ dissolved in $\mathrm{HI} / \mathrm{H}_{3} \mathrm{PO}_{2}\left(4 \mathrm{ml}\right.$ for $\mathrm{HI}$ and $0.8 \mathrm{ml}$ for $\left.\mathrm{H}_{3} \mathrm{PO}_{2}\right)$ mix solution. Bring to a boil in an oil bath until completely dissolved into a bright yellow transparent solution. The solution was cooled to $125^{\circ} \mathrm{C}$ for $15 \mathrm{~min}$ to allow (F-PEA) ${ }_{2} \mathrm{PbI}_{4}$ PSCs to crystal completely. After being naturally cooled to room temperature, the yellow (F-PEA) ${ }_{2} \mathrm{PbI}_{4}$ PSCs were filtered and washed with hexane. The (PEA) ${ }_{2} \mathrm{PbI}_{4}$ single crystals were grown with the same protocol.

Laser shocking (F-PEA) Pbl $_{\mathbf{4}}$ PSCs. A Diode-Pumped Femtosecond Industrial Laser $(1064 \mathrm{~nm}$ wavelength, repetition rate $7.5-375 \mathrm{kHz}$ and pulse duration of 280 fs-10 ps) was used as a laser energy source. A glass slide was used as a confining media because of its high shock impedance. A sacrificial coating was deposited by spraying a graphite ablator above the $10 \mu \mathrm{m}$ aluminum foil. The graphite layer was instantaneously ablated by a highly focused femtosecond laser (beam size 7-100 $\mu \mathrm{m})$, which created a shock wave that exerted enormous pressure on (F-PEA) ${ }_{2} \mathrm{PbI}_{4}$ PSCs and changed its lattice structure. The femtosecond laser scans are performed at a speed of $50 \mathrm{~mm} / \mathrm{s}$, with a pulse energy of $0-80 \mathrm{uJ}$.

Material characterization. The morphology and energy-dispersive X-ray spectroscopy (EDS) of (F-PEA) ${ }_{2} \mathrm{PbI}_{4}$ PSCs were obtained by field emission scanning electron microscopy (FE-SEM, Zeiss SIGMA). Atomic force microscope (AFM, SPA 400BRUKER Dimension Edge) was used to perform the perovskite morphology. PE Lambd 950 was used to perform the ultraviolet-visible absorption (UV-Vis) of a single crystal to obtain the optical bandgap of the film. Steady-state photoluminescence and temperature-dependent photoluminescence of perovskite single crystals were characterized by FLS 980 for laser excitation at $315 \mathrm{~nm}$. NICOLET 5700 Fourier transform infrared (FTIR) Spectrometer was used to characterization perovskite Molecular vibrations. Time-resolved photoluminescence (TRPL) data of single-crystal perovskite were collected by FLS1000 using $340 \mathrm{~nm}$ laser excitation. Photoluminescence quantum yield (PLQY) of the (F$\mathrm{PEA})_{2} \mathrm{PbI}_{4}$ PSCs were measured using an integrating sphere in the air under 
ambient conditions, and a laser with wavelength $340 \mathrm{~nm}$ was used as the excitation source. The crystal structure of (F-PEA) ${ }_{2} \mathrm{PbI}_{4}$ PSCs was obtained by Bruker D8 Venture. X-ray photoelectron spectroscopy (XPS) and ultraviolet photoelectron spectroscopy (UPS) data were measured by Thermo Fisher Scientific ESCALAB250Xi. Thermogravimetric analysis (TGA) and differential scanning calorimetry (DSC) of 2D single-crystal perovskite have an important application in thermal stability analysis of perovskite by using Mettler-Toledo TGA2 and DSC3, respectively. The thickness was measured using a surface profilometer (KLATencor D-120). Photocurrent measurement was carried out on the (F-PEA) ${ }_{2} \mathrm{PbI}_{4}$ PSCs photodetector with the structure of $\mathrm{Au} /(\mathrm{F}-\mathrm{PEA})_{2} \mathrm{PbI}_{4} / \mathrm{Au}$, which was measured by Keysight B2901A oscilloscope under $355 \mathrm{~nm}$ laser radiation (Power density, $0.16 \mathrm{~mW} / \mathrm{cm}^{2}$ ). The Au-wire with a thickness of $200 \mathrm{~nm}$, a length of 500 $\mu \mathrm{m}$ and $20 \mu \mathrm{m}$ in the gap, was used as the electrode with an effective area of $5 \times 10^{-2} \mathrm{~mm}^{2}$

Density functional theory simulation. The first-principles calculations were performed using the plane-wave pseudopotential method within the frameworks of DFT as implemented in the Vienna Ab initio Simulation Package ${ }^{54}$. The generalized gradient approximation Perdew-Burke-Ernzerhof (PBE) exchangecorrelation functional was used ${ }^{41}$. Band structure of (F-PEA $)_{2} \mathrm{PbI}_{4}$ PSCs was carried out along the symmetrical point $\mathrm{D}(-0.5,0,0.5), \mathrm{G}(0,0,0), \mathrm{Y}(0,0.5,0)$, B $(-0.5,0,0)$, and D. A $5 \times 5 \times 1$ Monkhorst-Pack k-point mesh for electronic Brillouin zone integration of $2 \mathrm{D}$ perovskites single crystal with cutoff energy 500 $\mathrm{eV}$.

Calculations. The max pressure of laser shocking was calculated by ${ }^{30}$ :

$$
P(\mathrm{~T})=\left[\frac{m I \alpha}{2 T(\alpha+1)}\right]^{1 / 2}
$$

Where $T$ is the pulse duration of the femtosecond laser, $I$ represent laser energy density of one pule (in $\mathrm{GW} / \mathrm{cm}^{2}$ ), $\alpha$ is a parameter of the reflected laser capability of the ablation layer ( $\alpha=0.1$ in graphite). The corresponding laser ablation mass is expressed in terms of $m$, which is determined by the mass change before and after the femtosecond laser. Simultaneously, we used a slightly excessive amount of graphite to avoid aluminum foil interference in the mass calculation.

We quantified the straining of (F-PEA) ${ }_{2} \mathrm{PbI}_{4}$ PSCs under laser shocking pressure by fitting the PXRD peak broadening, as follow: ${ }^{44}$

$$
\Delta d_{\varepsilon}=\sqrt{\Delta d_{\mathrm{obs}}^{2}-\Delta d_{\mathrm{int}}^{2}}=C_{\varepsilon} d
$$

where $\Delta d_{\text {obs }}{ }^{2}$ is the difference in d-spacing at full-width at half-maximum (FWHM) for a given plane. $\Delta d_{\text {int }}^{2}$ contribution from the instrument and $d$ is interplanar spacing.

TRPL spectra of (F-PEA) ${ }_{2} \mathrm{PbI}_{4}$ PSCs floating on the glass were fitted by 2constant exponential decay function: ${ }^{55}$

$$
f(t)=\mathrm{A}_{1} \exp \left(-\frac{t}{\tau_{1}}\right)+\mathrm{A}_{2} \exp \left(-\frac{t}{\tau_{2}}\right)+\mathrm{B}
$$

where $\mathrm{A}$ is the decay amplitude, $\tau_{1}$ and $\tau_{2}$ are carrier lifetimes, corresponding to extraction time and radiative recombination lifetime.

\section{Data availability}

The data that support the findings of this study are available on reasonable request from the corresponding author.

Received: 1 May 2021; Accepted: 19 July 2021;

Published online: 12 August 2021

\section{References}

1. Jeong, M. et al. Stable perovskite solar cells with efficiency exceeding $24.8 \%$ and $0.3-\mathrm{V}$ voltage loss. Science 369, 1615-1620 (2020).

2. Min, H. et al. Efficient, stable solar cells by using inherent bandgap of $\alpha$-phase formamidinium lead iodide. Science 366, 749-753 (2019).

3. Jiang, Q. et al. Surface passivation of perovskite film for efficient solar cells. Nat. Photonics 13, 460-466 (2019).

4. Wei, W. J. et al. Regulating second-harmonic generation by van der waals interactions in two-dimensional lead halide perovskite nanosheets. J. Am. Chem. Soc. 141, 9134-9139 (2019).

5. Fang, H.-H. et al. Unravelling light-induced degradation of layered perovskite crystals and design of efficient encapsulation for improved photostability. $A d v$. Funct. Mater. 28, 1800305 (2018).

6. Zhang, C. et al. Efficient stable graphene-based perovskite solar cells with high flexibility in device assembling via modular architecture design. Energy Environ. Sci. 12, 3585-3594 (2019).
7. Chu, Z. et al. Large cation ethylammonium incorporated perovskite for efficient and spectra stable blue light-emitting diodes. Nat. Commun. 11, 4165 (2020).

8. Tsai, H. et al. A sensitive and robust thin-film $\mathrm{x}$-ray detector using 2D layered perovskite diodes. Sci. Adv. 6, eaay0815 (2020).

9. Qin, C. et al. Stable room-temperature continuous-wave lasing in quasi-2D perovskite films. Nature 585, 53-57 (2020).

10. Ahmad, S. et al. Dion-Jacobson phase 2D layered perovskites for solar cells with ultrahigh stability. Joule 3, 794-806 (2019).

11. Grancini, G. et al. One-Year stable perovskite solar cells by 2D/3D interface engineering. Nat. Commun. 8, 15684 (2017).

12. Blancon, J. C. et al. Extremely efficient internal exciton dissociation through edge states in layered 2D perovskites. Science 355, 1288-1292 (2017).

13. Wei, Y. et al. Reverse-graded 2D ruddlesden-popper perovskites for efficient air-stable solar cells. Adv. Energy Mater. 9, 1900612 (2019).

14. Ummadisingu, A. et al. The effect of illumination on the formation of metal halide perovskite films. Nature 545, 208-212 (2017).

15. Kumar, A. et al. Excitons in 2D perovskites for ultrafast terahertz photonic devices. Sci. Adv. 6, eaax8821 (2020).

16. Straus, D. B. et al. Tailoring hot exciton dynamics in $2 \mathrm{~d}$ hybrid perovskites through cation modification. ACS Nano 14, 3621-3629 (2020).

17. Guo, S. et al. Pressure-suppressed carrier trapping leads to enhanced emission in two-dimensional perovskite $(\mathrm{HA})_{2}(\mathrm{GA}) \mathrm{Pb}_{2} \mathrm{I}_{7}$. Angew. Chem. Int. Ed. 59, 2-9 (2020).

18. Yin, T. et al. Pressure-engineered structural and optical properties of twodimensional $\left(\mathrm{C}_{4} \mathrm{H}_{9} \mathrm{NH}_{3}\right)_{2} \mathrm{PbI}_{4}$ perovskite exfoliated nm-thin flakes. J. Am. Chem. Soc. 141, 1235-1241 (2019).

19. Liu, G. et al. Pressure-induced bandgap optimization in lead-based perovskites with prolonged carrier lifetime and ambient retainability. Adv. Funct. Mater. 27, 1604208 (2017)

20. Xiao, G. et al. Pressure effects on structure and optical properties in cesium lead bromide perovskite nanocrystals. J. Am. Chem. Soc. 139, 10087-10094 (2017).

21. Montross, C. Laser shock processing and its effects on microstructure and properties of metal alloys: a review. Int. J. Fatigue 24, 1021-1036 (2002).

22. Millot, M. et al. Nanosecond $\mathrm{x}$-ray diffraction of shock-compressed superionic water ice. Nature 569, 251-255 (2019).

23. Bardy, S. et al. Numerical study of laser ablation on aluminum for shock-wave applications: development of a suitable model by comparison with recent experiments. Opt. Eng. 56, 011014 (2016).

24. Song, C., Tong, L., Liu, F., Ye, L. \& Cheng, G. J. Addressing the reliability and electron transport kinetics in halide perovskite film via pulsed laser engineering. Adv. Funct. Mater. 30, 1906781 (2019).

25. Gao, H. et al. Nanolithography. Large-scale nanoshaping of ultrasmooth 3D crystalline metallic structures. Science 346, 1352-1356 (2014).

26. Motlag, M. et al. Asymmetric 3d elastic-plastic strain-modulated electron energy structure in monolayer graphene by laser shocking. Adv. Mater. 31, e1900597 (2019).

27. Qiu, G. et al. Ultrafast laser-shock-induced confined metaphase transformation for direct writing of black phosphorus thin films. Adv. Mater. 30, 1704405 (2018).

28. Fang, Y. et al. Pressure-induced emission (PIE) and phase transition of a twodimensional halide double perovskite $(\mathrm{BA})_{4} \mathrm{AgBiBr}_{8}\left(\mathrm{BA}=\mathrm{CH}_{3}\left(\mathrm{CH}_{2}\right)_{3} \mathrm{NH}_{3}{ }^{+}\right)$. Angew. Chem. Int. Ed. 58, 15249-15253 (2019).

29. Fairand, B. P. \& Clauer, A. H. Laser generation of high-amplitude stress waves in materials. J. Appl. Phys. 50, 1497-1502 (1979).

30. Fabbro, R., Fournier, J., Ballard, P., Devaux, D. \& Virmont, J. Physical study of laser-produced plasma in confined geometry. J. Appl. Phys. 68, 775-784 (1990).

31. Bolger, J. A., Montross, C. S. \& Rode, A. V. Shock waves in basalt rock generated with high-powered lasers in a confined geometry. J. Appl. Phys. 86, 5461-5466 (1999).

32. Ballard, P., Fournier, J., Fabbro, R. \& Frelat, J. Residual-stresses induced by laser-shocks. J. Phys. III 1, 487-494 (1991).

33. Saidaminov, M. I. et al. Suppression of atomic vacancies via incorporation of isovalent small ions to increase the stability of halide perovskite solar cells in ambient air. Nat. Energy 3, 648-654 (2018).

34. Chen, Y. et al. Strain engineering and epitaxial stabilization of halide perovskites. Nature 577, 209-215 (2020).

35. Motlag, M. et al. Molecular-scale nanodiamond with high-density color centers fabricated from graphite by laser shocking. Cell Rep. Phys. Sci. 1, 100054 (2020).

36. Li, H. et al. Unusual pressure-driven phase transformation and band renormalization in $2 \mathrm{~d} v \mathrm{vdw}$ hybrid lead halide perovskites. Adv. Mater. 32, e1907364 (2020).

37. Wang, Y. et al. Pressure-induced phase transformation, reversible amorphization, and anomalous visible light response in organolead bromide perovskite. J. Am. Chem. Soc. 137, 11144-11149 (2015). 
38. Jaffe, A., Lin, Y., Mao, W. L. \& Karunadasa, H. I. Pressure-induced metallization of the halide perovskite $\left(\mathrm{CH}_{3} \mathrm{NH}_{3}\right) \mathrm{PbI}_{3}$. J. Am. Chem. Soc. 139, 4330-4333 (2017).

39. Kim, G. et al. Impact of strain relaxation on performance of $a$-formamidinium lead iodide perovskite solar cells. Science 370, 108-112 (2020).

40. $\mathrm{Li}, \mathrm{H}$. et al. Sensitive and stable $2 \mathrm{D}$ perovskite single-crystal $\mathrm{x}$-ray detectors enabled by a supramolecular anchor. Adv. Mater. 32, e2003790 (2020).

41. Zheng, $X$. et al. Managing grains and interfaces via ligand anchoring enables 22.3\%-efficiency inverted perovskite solar cells. Nat. Energy 5, 131-140 (2020).

42. Kikuchi, K., Takeoka, Y., Rikukawa, M. \& Sanui, K. Structure and optical properties of lead iodide based two-dimensional perovskite compounds containing fluorophenethylamines. Curr. Appl. Phys. 4, 599-602 (2004).

43. Kapil, G. et al. Strain relaxation and light management in tin-lead perovskite solar cells to achieve high efficiencies. ACS Energy Lett. 4, 1991-1998 (2019).

44. Zheng, X. et al. Improved phase stability of formamidinium lead triiodide perovskite by strain relaxation. ACS Energy Lett. 1, 1014-1020 (2016).

45. Grancini, G. \& Nazeeruddin, M. K. Dimensional tailoring of hybrid perovskites for photovoltaics. Nat. Rev. Mater. 4, 4-22 (2018).

46. Wang, B. et al. The charge carrier dynamics, efficiency and stability of twodimensional material-based perovskite solar cells. Chem. Soc. Rev. 48 , 4854-4891 (2019).

47. Righetto, M. et al. Hot carriers perspective on the nature of traps in perovskites. Nat. Commun. 11, 2712 (2020).

48. Wu, K. et al. Temperature-dependent excitonic photoluminescence of hybrid organometal halide perovskite films. Phys. Chem. Chem. Phys. 16, 22476-22481 (2014)

49. Long, $\mathrm{H}$. et al. Exciton-phonon interaction in quasi-two dimensional layered $(\mathrm{PEA})_{2}(\mathrm{CsPbBr})_{\mathrm{n}-1} \mathrm{PbBr}_{4}$ perovskite. Nanoscale 11, 21867-21871 (2019).

50. Savenije, T. J. et al. Thermally activated exciton dissociation and recombination control the carrier dynamics in organometal halide perovskite. J. Phys. Chem. Lett. 5, 2189-2194 (2014).

51. Liang, Y. et al. Broadband emission enhancement induced by self-trapped excited states in one-dimensional $\mathrm{EAPbI}_{3}$ perovskite under pressure. J. Phys. Chem. C. 124, 8984-8991 (2020).

52. Qian, Y. et al. A promising phase change material with record high ionic conductivity over a wide temperature range of a plastic crystal phase and magnetic thermal memory effect. ACS Appl. Mater. Interfaces $\mathbf{1 2}$ 28129-28138 (2020).

53. Wang, L. et al. A Eu${ }^{3+}-\mathrm{Eu}^{2+}$ ion redox shuttle imparts operational durability to $\mathrm{Pb}-\mathrm{I}$ perovskite solar cells. Science 363, 265-270 (2019).

54. Ren, H. et al. Efficient and stable ruddlesden-popper perovskite solar cell with tailored interlayer molecular interaction. Nat. Photonics 14, 154-163 (2020).

55. Byranvand, M. M. et al. P-type CuI islands on $\mathrm{TiO}_{2}$ electron transport layer for a highly efficient planar-perovskite solar cell with negligible hysteresis Adv. Energy Mater. 8, 1702235 (2018).

\section{Acknowledgements}

We acknowledge the support from The Institute of Technological Sciences, Wuhan University, and School of Industrial Engineering, Purdue University.

\section{Author contributions}

C.S. and G.C. conceived ideas and concepts, and initiated the research. C.S. performed the experimental studies and simulation. C.S. and H.Y. fabricated the perovskite single crystal. F.L. participated in the discussion and management of the project. C.S. and G.C carried out the analysis. C.S. and G.C. co-wrote the paper and revised the paper. All authors read and commented on the paper.

\section{Competing interests}

The authors declare no competing interests.

\section{Additional information}

Supplementary information The online version contains supplementary material available at https://doi.org/10.1038/s41467-021-25140-2.

Correspondence and requests for materials should be addressed to G.J.C.

Peer review information Nature Communications thanks the anonymous reviewer(s) for their contribution to the peer review of this work.

Reprints and permission information is available at http://www.nature.com/reprints

Publisher's note Springer Nature remains neutral with regard to jurisdictional claims in published maps and institutional affiliations.

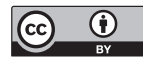

Open Access This article is licensed under a Creative Commons Attribution 4.0 International License, which permits use, sharing, adaptation, distribution and reproduction in any medium or format, as long as you give appropriate credit to the original author(s) and the source, provide a link to the Creative Commons license, and indicate if changes were made. The images or other third party material in this article are included in the article's Creative Commons license, unless indicated otherwise in a credit line to the material. If material is not included in the article's Creative Commons license and your intended use is not permitted by statutory regulation or exceeds the permitted use, you will need to obtain permission directly from the copyright holder. To view a copy of this license, visit http://creativecommons.org/ licenses/by/4.0/.

(C) The Author(s) 2021 